\title{
44. Plastische Durchbiegung von Stahlbetonbalken
}

\author{
Von Shizuo BAN \\ Abteilung für Architektur, Kyoto Universität, Kyoto \\ (Comm. by Y. TanakA, M.J.A., March 12, 1956)
}

Um ein statisch unbestimmtes Tragwerk bruchsicher zu machen, wird neuerdings das plastische Berechnungsverfahren angewandt. Dabei ist wesentlich, dass die Drehung des einzelnen plastischen Gelenks keinesfalls ihre eigene Drehfähigkeit überschreiten soll, bevor nicht das letzte plastische Gelenk in Erscheinung getreten ist. Diese Bedingung ist bei Stahlbauten von vorne herein erfüllt, weil eine Erhärtung des Materials wieder nach Beendigung des Fliessprozesses eintritt. Bei Stahlbetonbauten ist das aber nicht der Fall. Bei gewöhnlicher Bewehrung tritt der Bruch in folgender Weise auf: Zuerst der Stahl wird mit der üblichen Rissbildung des Betons bis zur Streckgrenze beansprucht. Die Formänderung des Balkens nimmt mit dem Fliessen des Stahls allmählich zu. Schliesslich wird die Tragfähigkeit des Balkens durch den Zusammenbruch des Betons an der Druckzone erschöpft. Das Biegemoment beim Bruch ist etwas grösser als das beim Fliessanfang.

Als plastische Durchbiegung bezeichnet man die Zunahme der Durchbiegung während des Fliessens. Sie hängt im Wesentlichen von dem Bewehrungsfaktor ab und kann sogar beim überbewehrten Balken verschwinden. Da das Drehvermögen des plastischen Gelenks in direkter Abhängigkeit von der plastischen Durchbiegung ist, wird in dieser Arbeit die letzte theoretisch sowie versuchsmässich untersucht.

Um die plastische Durchbiegung rechnerisch zu bestimmen, ist es unumgänglich, den Zusammenhang zwischen Biegemoment und Winkeländerung pro Längeneinheit des Balkens zu bestimmen. Diese Beziehung lässt sich ohne weiteres ermitteln, unter der Voraussetzung, dass die Spannungs-Dehnungsinien der beiden Materialien gegeben sind. Um das Berechnungsverfahren auf jede SpannungsDehnungskurve anwenden zu können, schlägt der Verfasser ein graphisches Verfahren vor, in dem er die Integralkurve der Spannungs-Stauchungslinie des Betons benutzt. Die zu Grund gelegte Gleichung lautet

$$
p \sigma_{s}\left(\delta_{c}+\delta_{s}\right)=\int_{0}^{\delta_{c}} f(\delta) d \delta,
$$

worin 
$p$ der Bewehrungsfaktor,

$\sigma_{s}$ die Zugspannung des Stahles,

$\delta_{s}$ die Dehnung des Stahles,

$\delta_{c}$ die Randstauchung des Betons,

$\boldsymbol{\sigma}=\boldsymbol{f}(\delta)$ die Spannung des Betons im Allgemeinen

ist. Beim Fliessanfang sind $\sigma_{s}=\sigma_{y}$ und $\delta_{s}=\delta_{y}$ gegeben und somit wird $\delta_{c}$ bestimmt. Bei der Betonzerstörung, wo die Betonrandstauchung einen festen Wert z.B. 3\% erreicht hat und die Zugspannung des Stahles unverändert bleibt, also $\sigma_{s}=\sigma_{y}$, kann man die Dehnung des Stahles $\delta_{s}$ bestimmen. Das Verhältnis des Abstandes der Druckresultierenden von dem Druckrand zur Nullinienhöhe lässt sich aus der Beziehung

$$
s / x=1-\int_{0}^{\delta_{c}} \sigma \delta d \delta / \delta_{c} \int_{0}^{\delta_{c}} \sigma d \delta
$$

ermitteln; jedoch genügt es, den folgenden angenäherten Wert anzunehmen:

$$
\begin{array}{lll}
s / x=0.333 & \text { für } \quad \delta_{c}=0, \\
s / x=0.433 & \text { für } \quad \delta_{c}=3 \% .
\end{array}
$$

(Zwischenwerte sind geradlinig einzuschalten.)

Das Biegemoment ergibt sich aus

$$
M=p b h^{2} \sigma_{s} \begin{gathered}
\delta_{s}+(1-s / x) \delta_{c} \\
\delta_{s}+\delta_{c}
\end{gathered}
$$

Die dementsprechende Winkeländerung ist

$$
\varphi=\left(\delta_{c}+\delta_{s}\right) / h \text {. }
$$

Die Durchbiegung des Balkens einschliesslich der plastischen Durchbiegung im Sinne der vorherigen Definition lässt sich nach dem Mohr'schen Gesetz ermitteln. Nimmt man einen durch zwei Einzellasten symmetrisch belasteten Balken an und bezeichnet man mit $a$ die Entfernung der beiden Einzellasten, so erhält man angenähert

$$
\delta_{p}=\frac{a}{2}\left(\varphi_{\max }-\varphi_{y}\right) \frac{1}{2}\left(l-\frac{1}{2} a\right)
$$

wobei

$\delta_{p}$ die plastische Durchbiegung,

$l$ die Spannweite des Balkens,

$\varphi_{y} \mathrm{bzw} . \varphi_{\max }$ die Winkeländerung pro Längeneinheit des Balkens beim Fliessanfang bzw. bei der Betonzerstörung

ist. Die aus der Gleichung (5) berechnete plastische Durchbiegung ist stets kleiner als die tatsächliche und zwar desto kleiner, je geringer das Verhältnis $a / l$ ist. Diese Tatsache ist darauf zurückzuführen, dass die Spannung der Zugbewehrung nach der Rissbildung nicht mehr der Verteilung des Biegemoments folgen kann. Aus diesem Grunde dürfte es wohl richtig sein, die wirkliche Fliesstrecke 
länger anzunehmen als der Abstand der Einzellasten. Die Zusatzlänge der Fliesstrecke hängt vermutlich von der Balkenhöhe $a b$. Auf Grund der bisherigen Versuchsergebnisse wurde die tatsächliche Fliesstrecke nach jeder Seite um die Länge des Hebelarmes der Innenkraft grösser angenommen, was dazu führte, dass sich die plastische Durchbiegung angenähert aus nachstehender Gleichung berechnen lässt:

$$
\delta_{p}=\frac{1}{4}\left(\varphi_{\text {max }}-\varphi_{y}\right)\left(l-\frac{1}{2} a\right)(a+2 j)
$$

wobei $j$ der Hebelarm der Innenkraft ist.

Um die berechnete plastische Durchbiegung auch durch den Versuch zu bestätigen, führte der Verfasser eine Reihe von Versuchen durch. Der Versuch umfasste im ganzen 38 Balken. In der ersten Versuchsreihe ,, A“ wurden Versuche über den Einfluss des Bewehrungsfaktors angestellt, in ,, B“ über den Einfluss der Lage der Einzellasten, in , C “ über den Einfluss des Verhältnisses der Balkenhöhe zur Spannweite und in , $D$ “ über den Einfluss der Güte des Betors.

Bei der Berechnung wurde angenommen, dass der Stahl innerhalb der Streckgrenze dem Hooke'schen Gesetz folgt. Die Betonstauchungskurve wurde bis zur Höchstspannung durch Versuch festgestellt. Die daran anschliessende Teilkurve im plastischen Bereich wurde so bestimmt, dass sich die Betonspannung allmählich bis auf das 0.92-fache der Höchstspannung verkleinerte, wo die dazugehörige Höchststauchung 3\% beträgt. Der Vergleich der berechneten plastischen Durchbiegung mit der durch Versuch ermittelten lässt erkennen, dass die Abweichung der Versuchsergebnisse von den berechneten Durchbiegungen trotz recht erheblicher Verstreuchungen im Durchschnitt sehr klein ist. 